\title{
KALMAN BUCY FILTER BASED DATA ASSIMILATION WITH NAVY LAYERED OCEAN MODEL
}

\author{
Amir Asif \\ Computer Science, York University \\ North York, ON, Canada M3J 1P3 \\ Email: asif@cs.yorku.ca
}

\begin{abstract}
Computationally efficient implementations of the Kalman Bucy filter (KBf) for data assimilation are presented. We apply our algorithms to assimilate satellite altimetry data into the Navy Layered Ocean Model (NLOM). Compared to direct KBf, our implementations provide computational savings of up to two orders of magnitude of the linear dimension of the ocean field. Results from a $1 / 4^{\circ}$ equatorial channel simulation of the Pacific ocean are included.
\end{abstract}

\section{INTRODUCTION}

In physical oceanography, combining data with nonlinear state models based on the primitive Navier Stokes equation is referred to as data assimilation $[1,2]$. The objective is to estimate the state of the ocean circulation fields for example the sea surface height (SSH) and the ocean velocity components. The large dimensions and nonlinearity of the state models preclude the application of sophisticated signal processing algorithms like the Kalman Bucy filter (KBf) from data assimilation.

The paper presents practical implementations of the KBf, referred to as the local KBf [3], for estimating nonlinear ocean circulation fields. Unlike [3] where dynamical models arising from discretization of linear partial differential equations (pde) are used, we consider the more realistic Navy layered ocean model (NLOM) [4]. Another difference arises due to the difference in the structure of the state matrices. While the state matrices in [3] are block banded, the state matrices in the NLOM are full matrices. However, the blocks that constitute the state matrices are subblock banded so the local KBf is applied at the subblock level to the NLOM.

The paper is organized as follows. Section 2 describes the dynamical models and introduces the notation. Section 3 presents the data assimilation algorithm based on the KBf. Twin experiments are included in section 4 while section 5 concludes the paper.

\section{DYNAMICAL MODELS}

For compactness, the primitive pde's used to numerically compute the ocean circulation fields in the NLOM are expressed as

$$
\frac{\partial \psi}{\partial t}=\mathcal{D} \psi+\mathcal{F}, \quad \mathcal{D}=\sum_{n_{1}, n_{2}, n_{3}}\left(\frac{\partial}{\partial \theta} \frac{\partial}{\partial \phi} a_{n_{1} n_{2} n_{3}}(\theta, \phi)\right)
$$

where $\psi$ represents multiple $(q)$ fields like sea surface height (SSH) $h$, and the latitudinal and longitudinal velocity components $(u, v)$. The operator $\mathcal{D}$ is the pde operator, $\theta$ and $\phi$ are the latitudinal and longitudinal coordinates, and $\mathcal{F}$ incorporates wind forcings.
In a numerical simulation, the ocean domain constrained by the latitudinal and longitudinal boundaries is discretized into an $(I \times$ $J)$ grid with $L$ layers. A set of finite difference equations obtained by discretizing (1) computes the ocean circulation fields $(h, u, v)$ for each layer. In our derivations, a row of the discretized field in layer $\ell$ is first mapped into a $(J \times 1)$ random vector and these are stacked one on the top of the other to form a $(q I J \times 1)$ field vector

$$
\begin{gathered}
\Psi^{(\ell)}=[\underbrace{h_{1}^{T}, u_{1}^{T}, v_{1}^{T}}_{\Psi_{1}^{(\ell)}}, \underbrace{h_{2}^{T}, u_{2}^{T}, v_{2}^{T}}_{\Psi_{2}^{(\ell)}}, \ldots, \underbrace{h_{I}^{T}, u_{I}^{T}, v_{I}^{T}}_{\Psi_{I}^{(\ell)}}]^{T}, \text { where } \\
h_{i}=\left[h_{i 1}, \ldots, h_{i J}\right]^{T}, u_{i}=\left[u_{i 1}, \ldots, u_{i J}\right]_{,}^{T} v_{i}=\left[v_{i 1}, \ldots, v_{i J}\right]^{T},
\end{gathered}
$$

for $1 \leq i \leq I$ and $(1 \leq \ell \leq L)$. The state vector $\Psi$ consists of field vectors $\Psi^{(\ell)}$ for each layer stacked together in the order of the depth. Additional terms that provide coupling between the vertical layers are also included in the dynamical model of (1).

The covariance matrix $\mathcal{P}$ of the state vector $\Psi$ is expressed in terms of its constituent blocks as

$$
\mathcal{P}=\left\{P^{\left(\ell_{1} \ell_{2}\right)}\right\} \text { with } P^{\left(\ell_{1} \ell_{2}\right)}=\left[\begin{array}{ccc}
P_{11}^{\left(\ell_{1} \ell_{2}\right)} & \cdot & P_{1 I}^{\left(\ell_{1} \ell_{2}\right)} \\
\dot{\left.\ell_{1} \ell_{2}\right)} & \cdot & P_{I I}^{\left(\dot{\left.\ell_{1} \ell_{2}\right)}\right.}
\end{array}\right]
$$

where $P^{\left(\ell_{1} \ell_{2}\right)}$ is a $(q I J \times q I J)$ block that denotes the covariance between the lexicographic ordered vectors $\Psi^{\left(\ell_{1}\right)}$ and $\Psi^{\left(\ell_{2}\right)}$ for layers $\ell_{1}$ and $\ell_{2}$ of the ocean field. The covariance blocks $P^{\left(\ell_{1} \ell_{2}\right)}$ are further expressed in terms of $(q J \times q J)$ subblocks $P_{i j}^{\left(\ell_{1} \ell_{2}\right)}$ representing the covariance between the $q$ ocean fields of row $i$ in layer $\ell_{1}$ and the corresponding fields of row $j$ in layer $\ell_{2}$. Our data assimilation algorithm is expressed in terms of the $(q J \times q J)$ blocks $P_{i j}^{\left(\ell_{1} \ell_{2}\right)}$ for $(1 \leq i, j \leq I)$ and $\left(1 \leq \ell_{1}, \ell_{2} \leq L\right)$.

Satellite Measurements: The satellite tracks are curved, so altimetry data is available on a few points along $N$ adjacent rows, $m, \ldots, p$, in the top most layer $(\ell=1)$. The observation model is

$$
\begin{aligned}
& \underbrace{\left[\begin{array}{c}
\mathcal{Y}_{m}^{(1)}(k+1) \\
\vdots \\
\mathcal{Y}_{p}^{(1)}(k+1)
\end{array}\right]}_{\mathcal{Y}(k+1)}=\underbrace{\left[\begin{array}{lll}
\Theta(k+1) & \underline{0} & 0
\end{array}\right]}_{\mathcal{H}(k+1)} \Psi(k+1)+\xi(3) \\
& \text { with } \Theta(k+1)=\left[\begin{array}{ccccccccc}
\cdot & \underline{0} & \theta_{m} & \underline{0} & \cdot & \underline{0} & \underline{0} & \underline{0} & \cdot \\
\cdot & \cdot & \cdot & \cdot & \cdot & \cdot & \cdot & \cdot & \cdot \\
\cdot & \underline{0} & \underline{0} & \underline{0} & \cdot & \underline{0} & \theta_{p} & \underline{0} & \cdot
\end{array}\right] \text { (4) }
\end{aligned}
$$


For a total of $L$-vertical layers, the observation matrix $\mathcal{H}(k+1)$ is of dimensions $(q N J \times q L I J)$ and is highly sparse. The constituent blocks $\theta_{i}$ 's of $\Theta$ are also sparse since the satellite provides data only on the $\mathrm{SSH}, h$. The velocity components $(u, v)$ are not observed. The variable $\xi$ in (3) represents the measurement noise, assumed Gaussian with covariance $\mathcal{R}$, and $k$ is the time index.

\section{EXTENDED KBF}

To apply the KBf for data assimilation, we propose the extended filtering approach where a real-time linear approximation of the nonlinear dynamic model (1) is used as the state equation in the $\mathrm{KBf}$. The linearized state model for the extended KBf is obtained by approximating (1) with the shallow water model

$$
\begin{array}{r}
\frac{\partial h^{(\ell)}}{\partial t}+\frac{H^{(\ell)}}{a \cos \theta}\left[\frac{\partial u^{(\ell)}}{\partial \phi}+\frac{\partial\left(v^{(\ell)} \cos \theta\right)}{\partial \theta}\right]=0 \quad \text { (5) } \\
\frac{\partial u^{(\ell)}}{\partial t}-2 \Omega v^{(\ell)} \sin \theta+\sum_{\tau=1}^{L} \frac{h^{(\ell)} G^{(\ell \tau)}}{a \cos \theta} \frac{\partial\left(h^{(\tau)}-H^{(\tau)}\right)}{\partial \phi}=F_{\phi}(6) \\
\frac{\partial v^{(\ell)}}{\partial t}+2 \Omega u^{(\ell)} \sin \theta+\sum_{\tau=1}^{L} \frac{h^{(\ell)} G^{(\ell \tau)}}{a} \frac{\partial\left(h^{(\tau)}-H^{(\tau)}\right)}{\partial \theta}=F_{\theta}(7)
\end{array}
$$

where $H^{(\ell)}$ denotes the thickness of layer $\ell, \Omega$ is the Coriolis parameter, $\left(F_{\phi}, F_{\theta}\right)$ are the longitudinal and latitudinal components of the forcing term $F$, and $G^{(\ell \tau)}$ is the reduced gravity parameter

$$
G^{(\ell \tau)}= \begin{cases}g, & \tau \leq \ell \\ g\left(1-\frac{\rho^{(\tau)}-\rho^{(\ell)}}{\rho^{(0)}}\right), & \tau>l\end{cases}
$$

with $\rho^{(\ell)}$ the $\ell^{\prime}$ th layer density and $\rho^{(0)}$ the average background density. Equations (5)-(7) are discretized with the staggered Cgrid Leap Frog finite difference scheme, followed by a real-time Taylor approximation of the nonlinear terms. The state equation is

$$
\Psi(k+1)=\mathcal{A}(k) \Psi(k)+\mathcal{C}(k) W(k) .
$$

where the state matrices $\mathcal{A}$ and $\mathcal{C}$ are full matrices that are partitioned in terms of the time varying blocks, $\mathcal{A}=\left\{A^{\left(\ell_{1} \ell_{2}\right)}\right\}$ and $\mathcal{C}=\left\{C^{\left(\ell_{1} \ell_{2}\right)}\right\}$, for $\left(1 \leq \ell_{1}, \ell_{2} \leq L\right)$. The blocks $A^{\left(\ell_{1} \ell_{2}\right)}$ and $C^{\left(\ell_{1} \ell_{2}\right)}$ are of the same dimensions as the covariance blocks $P^{\left(\ell_{1} \ell_{2}\right)}$ but are highly structured with $A^{\left(\ell_{1} \ell_{2}\right)}$ being $M_{1}$-block banded and $B^{\left(\ell_{1} \ell_{2}\right)}$ being $M_{2}$-block banded. This block banded structure arises from the local interactions of the fields resulting from the discretization of the pde's (5)-(7). The term $W(k)$ incorporates the wind forcings and has a covariance $\mathcal{Q}$.

Data Assimilation Algorithm: The linear dimension of the field $\Psi$ in a meaningful ocean circulation simulation is of the order of $10^{6}$ to $10^{9}$ elements. In such applications, implementation of the direct $\mathrm{KBf}$ requires storage and manipulation of covariance matrices of order $10^{6}$ to $10^{9}$. The inversion or multiplication of such matrices therefore requires up to $10^{27}$ flops, which is computationally not feasible. Based on theorem 1 [5], we derive a nearoptimal implementation of the $\mathrm{KBf}$ in which a subset of subblocks $P_{i j}^{\left(\ell_{1} \ell_{2}\right)}$ of block $P^{\left(\ell_{1} \ell_{2}\right)}$ in $\mathcal{P}$ are updated during each iteration. Theorem 1 is explained next.

Theorem 1: Given blocks $\left\{P_{i j}^{\left(\ell_{1} \ell_{2}\right)}\right\}$ on the first $M$-subblock diagonals of $P^{\left(\ell_{1} \ell_{2}\right)}=\left(B^{\left(\ell_{1} \ell_{2}\right)}\right)^{-1}, B^{\left(\ell_{1} \ell_{2}\right)}$ being $M$-subblock banded, the subblocks outside the $M$-subblock diagonals in $P^{\left(\ell_{1} \ell_{2}\right)}$ are expressed as

$$
\begin{aligned}
& P_{i j}^{\left(\ell_{1} \ell_{2}\right)}=\left[\begin{array}{ccc}
P_{i i+1}^{\left(\ell_{1} \ell_{2}\right)} & \ldots & P_{i i+M}^{\left(\ell_{1} \ell_{2}\right)}
\end{array}\right] \\
& {\left[\begin{array}{ccc}
P_{i+1 i+1}^{\left(\ell_{1} \ell_{2}\right)} & \cdot & P_{i+1 i+M}^{\left(\ell_{1} \ell_{2}\right)} \\
& \ddots & \\
P_{i+M i+1}^{\left(\ell_{1} \ell_{2}\right)} & \cdot & P_{i+M i+M}^{\left(\ell_{1} \ell_{2}\right)}
\end{array}\right]^{-1}\left[\begin{array}{c}
P_{i+1 j}^{\left(\ell_{1} \ell_{2}\right)} \\
\vdots \\
P_{i+M j}^{\left(\ell_{1} \ell_{2}\right)}
\end{array}\right]}
\end{aligned}
$$

for $1 \leq i<(I-M)$ and $(i+M)<j \leq I$.

In the local KBf, we impose a $M$-subblock banded approximation on the inverse of block $P^{\left(\ell_{1} \ell_{2}\right)}$ in the covariance matrix $\mathcal{P}$. Only subblocks $P_{i j}^{\left(\ell_{1} \ell_{2}\right)}$ within the first $M$ block diagonals of $P^{\left(\ell_{1} \ell_{2}\right)}$ are updated. We refer to these subblocks as the significant block. Any subblocks other than the significant blocks, if required, is computed using theorem 1 . Below we briefly outline the data assimilation algorithm.

Local KBf: Initial conditions: $\widehat{\Psi}(0 \mid 0), \mathcal{P}(0 \mid 0), \mathcal{Q}, \mathcal{R}$ at $k=0$.

Step 1: Update the field $\widehat{\Psi}(k+1 \mid k)$ using the nonlinear NLOM, (1). Step 2: In terms of the constituent blocks of $\mathcal{A}=\left\{A^{\left(\ell_{1} \ell_{2}\right)}\right\}$, $\mathcal{C}=\left\{C^{\left(\ell_{1} \ell_{2}\right)}\right\}, \mathcal{P}=\left\{P^{\left(\ell_{1} \ell_{2}\right)}\right\}$, and $\mathcal{Q}=\left\{Q^{\left(\ell_{1} \ell_{2}\right)}\right\}$, the error covariance matrix is updated as

$$
\begin{aligned}
& P^{\left(\ell_{1} \ell_{2}\right)}(k+1 \mid k)= \\
& \sum_{\tau_{2}=1}^{L}\left(\sum_{\tau_{1}=1}^{L} A^{\left(\ell_{1} \tau_{1}\right)}(k) P^{\left(\tau_{1} \tau_{2}\right)}(k \mid k)\right)\left(A^{\left(\ell_{2} \tau_{2}\right)}(k)\right)^{T} \\
& +\sum_{\tau_{2}=1}^{L}\left(\sum_{\tau_{1}=1}^{L} C^{\left(\ell_{1} \tau_{1}\right)}(k) Q^{\left(\tau_{1} \tau_{2}\right)}(k)\right)\left(C^{\left(\ell_{2} \tau_{2}\right)}(k)\right)^{T}
\end{aligned}
$$

for $\left(1 \leq \ell_{1}, \ell_{2} \leq L\right)$. Eq. (11) includes sum of several product terms of the form, $A^{(* *)} P^{(* *)}(k \mid k)\left(A^{(* *)}\right)^{T}$ In order to expand (11) in terms of subblocks $P_{i j}^{\left(\ell_{1} \ell_{2}\right)}(k+1 \mid k)$, each product term $A^{(* *)} P^{(* *)}(k \mid k)\left(A^{(* *)}\right)^{T}$ is expressed in terms of the constituent subblocks as

$$
\begin{aligned}
& {\left[A_{1}^{(* *)} P_{i-1 j-1}^{(* *)}+A_{2}^{(* *)} P_{i j-1}^{(* *)}+A_{3}^{(* *)} P_{i+1 j-1}^{(* *)}\right]\left(A_{1}^{(* *)}\right)^{T} } \\
+ & {\left[A_{1}^{(* *)} P_{i-1 j}^{(* *)}+A_{2}^{(* *)} P_{i j}^{(* *)}+A_{3}^{(* *)} P_{i+1 j}^{(* *)}\right]\left(A_{2}^{(* *)}\right)^{T} } \\
+ & {\left[A_{1}^{(* *)} P_{i-1 j+1}^{(* *)}+A_{2}^{(* *)} P_{i j+1}^{(* *)}+A_{3}^{(* *)} P_{i+1 j+1}^{(* *)}\right]\left(A_{3}^{(* *)}\right)^{T} }
\end{aligned}
$$

where the subblocks $P_{* *}^{(* *)}$ are computed at $(k \mid k)$; the time indexes are omitted due to space constraints. In deriving (12), we have assumed that the block $A^{\left(\ell_{1} \ell_{2}\right)}$ is tridiagonal subblock banded ${ }^{1}$, $\left(M_{1}=1\right)$, with subblocks $A_{1}^{\left(\ell_{1} \ell_{2}\right)}, A_{2}^{\left(\ell_{1} \ell_{2}\right)}$, and $A_{3}^{\left(\ell_{1} \ell_{2}\right)}$ on its lower, main, and upper subblock diagonals. The $M$-subblock banded approximation on block $P^{\left(\ell_{1} \ell_{2}\right)}(k+1 \mid k)$ implies that we update only the main and the first $M$ upper subblock diagonals in each such block. In other words, we compute subblocks $P_{i j}^{\left(\ell_{1} \ell_{2}\right)}(k+$ $1 \mid k),|j-i| \leq M$, in $P^{\left(\ell_{1} \ell_{2}\right)}$ using (11)-(12). From (12), an update $P_{i j}^{\left(\ell_{1} \ell_{2}\right)}(k+1 \mid k)$ requires subblocks

$$
P_{i j}^{\left(\ell_{1} \ell_{2}\right)}(k \mid k), \quad \text { for }|j-i| \leq(M+2)
$$

${ }^{1}$ The discussion presented here is generalizable to the $M_{1}$-subblock banded case for the state matrix $\mathcal{A}$ 
from the last iteration of the KBf. Of the required subblocks $P_{i j}^{\left(\ell_{1} \ell_{2}\right)}$ at $(k \mid k)$ in (13), the subblocks on the first $M$ subblock diagonals are obtained from the previous iteration of the KBf. The remaining subblocks on diagonals $(M+1)$ and $(M+2)$ are computed from the first $M$-subblock diagonals using theorem 1. A similar procedure is used to approximate $Q^{\left(\ell_{1} \ell_{2}\right)}$.

Step 3: Compute the Kalman gain, which in terms of blocks is

$$
\begin{gathered}
K^{(\ell)}(k+1)=P^{(\ell 1)}(k+1 \mid k) \Theta^{T}(k+1) . \\
{\left[\Theta(k+1) P^{(11)}(k+1 \mid k) \Theta^{T}(k+1)+R^{(11)}(k+1)\right]^{-1}}
\end{gathered}
$$

where $K^{(\ell)}$ is the $(q I J \times q N J)$ Kalman gain for layer $\ell,(1 \leq$ $\ell \leq L)$. At the subblock level, (14) is

$$
\begin{aligned}
& K_{i}^{(\ell)}(k+1)=\left[\begin{array}{ccc}
P_{1 m}^{(\ell 1)} \theta_{m}^{T} & \ldots & P_{1 p}^{(\ell 1)} \theta_{p}^{T}
\end{array}\right] . \\
& {\left[\begin{array}{ccc}
\left(\theta_{m} P_{m m}^{(11)} \theta_{m}^{T}+R_{m m}^{(11)}\right) & \cdot & \theta_{m} P_{m p}^{(11)} \theta_{p}^{T} \\
\theta_{p} P_{p m}^{(11)} \theta_{m}^{T} & \ddots & \left(\theta_{p} P_{p p}^{(11)} \theta_{p}^{T}+R_{m m}^{(11)}\right)
\end{array}\right]}
\end{aligned}
$$

where $K_{i}^{(\ell)}(k+1)$ is a $(q J \times q N J)$ subblock that represents the Kalman gain for row $i$ of layer $\ell,(1 \leq i \leq I)$ and $(1 \leq \ell \leq L)$. To update the Kalman gain $K_{i}^{(\ell)}(k+1)$ using (15), only a few of the required subblocks $P_{i j}^{\left(\ell_{1} \ell_{2}\right)}$ lie outside the $M$-subblock diagonals in block $P^{\left(\ell_{1} \ell_{2}\right)}$. These are computed from the subblocks within the $M$-subblock diagonal of $P^{\left(\ell_{1} \ell_{2}\right)}$ using theorem 1 .

Step 4: Compute the predicted estimate from the measurements. At the block level,

$$
\begin{aligned}
& \widehat{\Psi}^{(\ell)}(k+1 \mid k+1)=\widehat{\Psi}^{(\ell)}(k+1 \mid k) \\
& \quad+K^{(\ell)}(k+1)\left[\mathcal{Y}^{(1)}(k+1)-\Theta(k+1) \widehat{\Psi}_{m}^{(1)}(k+1 \mid k)\right]
\end{aligned}
$$

for $(1 \leq \ell \leq L)$. Expanding further to the subblock level gives

$$
\begin{aligned}
\widehat{\Psi}_{i}^{(\ell)}(k+1 \mid k+1)= & \widehat{\Psi}_{i}^{(\ell)}(k+1 \mid k) \\
+K_{i}^{(\ell)}(k+1) \cdot & {\left[\begin{array}{c}
\mathcal{Y}_{m}^{(1)}(k+1)-\theta_{m} \widehat{\Psi}_{m}^{(1)}(k+1 \mid k) \\
\vdots \\
\mathcal{Y}_{p}^{(1)}(k+1)-\theta_{p} \widehat{\Psi}_{p}^{(1)}(k+1 \mid k)
\end{array}\right], }
\end{aligned}
$$

for $(1 \leq i \leq I)$ and $(1 \leq \ell \leq L)$. The ocean field $\Psi$ is updated one row at a time.

Step 5: Compute the a posteriori covariance matrix using

$$
\begin{aligned}
& P^{\left(\ell_{1} \ell_{2}\right)}(k+1 \mid k+1)=P^{\left(\ell_{1} \ell_{2}\right)}(k+1 \mid k) \\
& -K^{\left(\ell_{1}\right)}(k+1) \Theta(k+1) P^{\left(1 \ell_{2}\right)}(k+1 \mid k)
\end{aligned}
$$

for $\left(1 \leq \ell_{1}, \ell_{2} \leq L\right)$. Simplifying the above expression to the subblock level gives

$$
\begin{array}{r}
P_{i j}^{\left(\ell_{1} \ell_{2}\right)}(k+1 \mid k+1)=P_{i j}^{\left(\ell_{1} \ell_{2}\right)}(k+1 \mid k) \\
-K_{1}^{\left(\ell_{1}\right)}(k+1)\left[\begin{array}{c}
\theta_{m} P_{m j}^{\left(1 \ell_{2}\right)}(k+1 \mid k) \\
\vdots \\
\theta_{p} P_{p j}^{\left(1 \ell_{2}\right)}(k+1 \mid k)
\end{array}\right]
\end{array}
$$

for $(1 \leq i, j \leq I),|j-i| \leq M$, and $\left(1 \leq \ell_{1}, \ell_{2} \leq L\right)$. Eq. (20) is updated for $P_{i j}^{\left(\ell_{1} \ell_{2}\right)}(k+1 \mid k+1)$ that lie within the first $M$ subblock diagonals. Some subblocks $P_{i j}^{\left(\ell_{1} \ell_{2}\right)}(k+1 \mid k)$ required in (20) lie outside the first $M$ subblock diagonals. These outside subblocks are computed from the subblock lying within the $M$ subblock diagonals using theorem 1 .

Step 6: Increment $k$ by 1 and go back to step 1 .

\section{TWIN EXPERIMENT}

To test our data assimilation scheme, an equatorial channel of the Pacific ocean within the latitudes of $\pm 20^{\circ}$ and the longitudes of $306^{\circ}$ to $374^{\circ}$ is simulated. Twin experiments on a $1 / 4^{\circ}, 2 \frac{1}{2}$ hydrodynamic NLOM with analytical winds $\left(F_{\phi}, F_{\theta}\right)$ are performed. The dimension of the state vector is roughly of $\mathrm{O}\left(10^{6}\right)$.

Deterministic setup: runs the NLOM with analytical winds to propagate the deterministic components. Such theoretical estimates are often used by oceanographers to model ocean circulation in various regions of the ocean.

Real world setup: simulates the real conditions by extending the deterministic setup with addition of White Gaussian noise with a signal to noise ratio (SNR) of $12 \mathrm{~dB}$ to the wind forcings $\left(F_{\phi}, F_{\theta}\right)$. The two simulations described above are run for a simulated period of 6 months. The resulting fields $(h, u, v)$ provide the initial fields for our twin experiment.

Data Assimilation: The output of the real world setup is assimilated as pseudo data, (3), into the deterministic setup to estimate the results of the real world. Gaussian noise of $10 \mathrm{~dB}$ is added to the altimetry observations, $h$.

Results: In our data assimilation experiment, we use a pentadiagonal subblock approximation $(M=2)$ in the local KBf. Figs. 1, 2 , and 3 show estimates of the sea surface height ( $\mathrm{SSH}$ ) for the top layer from the three setups described earlier, at day 36 after three satellite repeat cycles. Both mesh and contour plots are shown. A visual comparison of the figures illustrates that the SSH image associated with the satellite scanned sparse data (fig. 3) is a better estimate of the actual ocean state condition (fig. 1), in our case, the real world run, than the field predicted with no data assimilation (fig. 2). The latter is a low pass version of the actual SSH. The data assimilated SSH incorporates the finer details like the eddies as, for example, the valleys and the peaks in fig. 1 are well reproduced in fig. 3 but are smoothed out in fig. 2. In fig. 4, a quantitative comparison based on the mean square error (MSE) between different frames of the SSH is performed. The relative MSE (RMSE) plotted in fig. 4, is defined as the ratio of the MSE of the data assimilated run to the MSE of the deterministic run. The region below ordinate $(y=1)$ represents improvement made by the data assimilated run. With time, the RMSE's for both layers drop suggesting improvements with the data assimilated prediction. The improvement is higher, roughly $25 \%$ after 36 days, for the upper layer for which altimetry data is available. No data is assimilated for layer 2, yet we see an improvement of about $10 \%$. Our KBf implementation successfully correlates the two layers and projects the surface information into the lower layer.

\section{SUMMARY}

Assimilation of the altimetry data into the NLOM has been investigated. We use a local implementation of the KBf that couples dynamic linearization with a $M$-block banded approximation to the inverse of the error covariance matrix in the KBf. Results from twin experiments on a $1 / 4^{\circ}, 2 \frac{1}{2}$ layer hydrodynamic NLOM show 

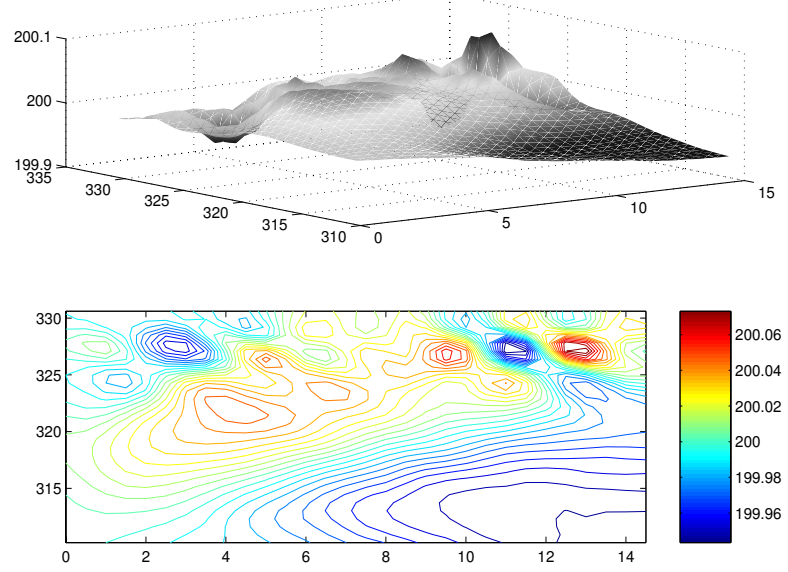

Fig. 1. Mesh and contour plots of the sea surface height (SSH) in meters for the top layer from the real world setup. The scale is shown in colour on the right of the contour plot.
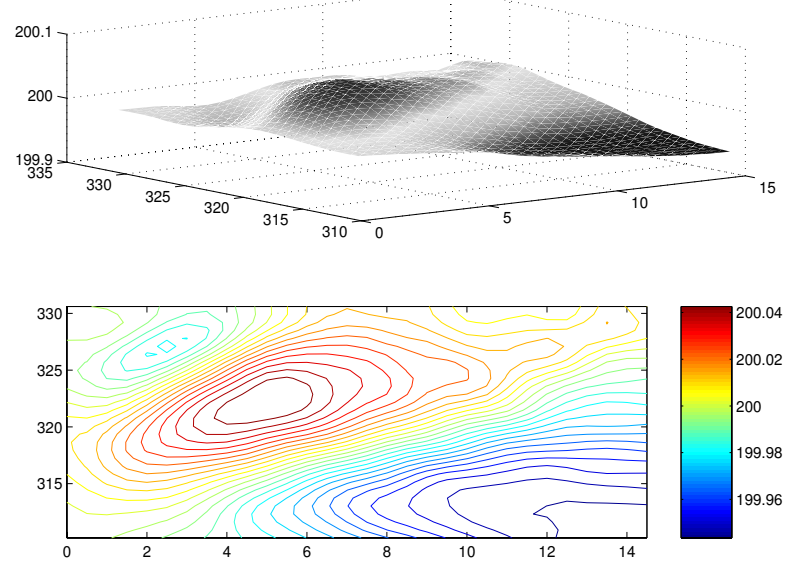

Fig. 2. Mesh and contour plots of the SSH of the top layer from the deterministic setup. The plots do not capture the finer details like the eddies, the troughs, and the crests of fig. 1.

significant improvement in the reconstruction of the ocean circulation fields. Although we assimilate altimetry data for only the upper layer, reconstructions of both upper and lower layers are improved. The KBf implementation projects the surface information into the subsurface layer.

\section{REFERENCES}

[1] T. M. Chin, A. J. Mariano, and E. P. Chassignet, "Spatial Regression with Markov Random Fields for Kalman Filter Approximation in Least Squares Solution of Oceanic Data Assimilation Problems," Journal of Geophysical Research, vol. 104, pp. 7991-8014, 1999.

[2] D. T. Pham, J. Verron, and M. .C. Roubaud, "A Singular Extended Kalman Filter for Data Assimilation in Oceanography," Journal of Marine Systems, vol. 16, pp. 323-340, 1998.
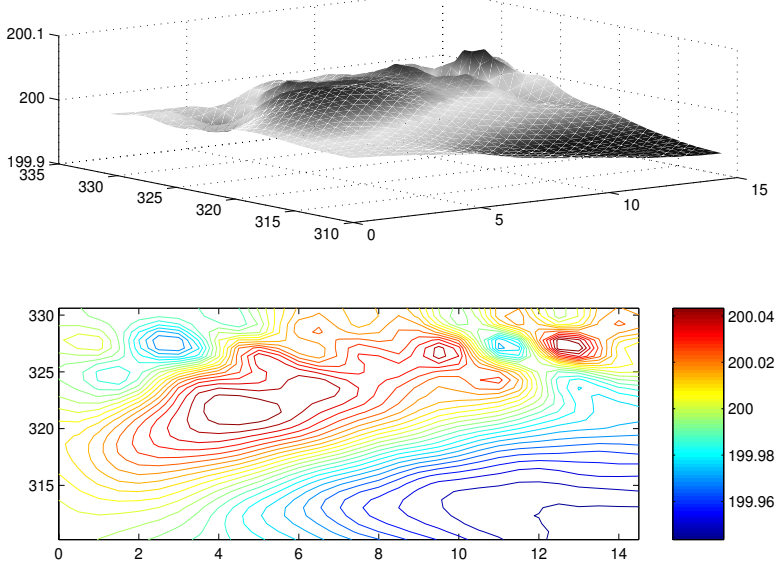

Fig. 3. Mesh and contour plots of the SSH of the top layer from the data assimilation setup. These images illustrate that the data assimilated SSH's are a better estimate of the real world run, fig. 1 .

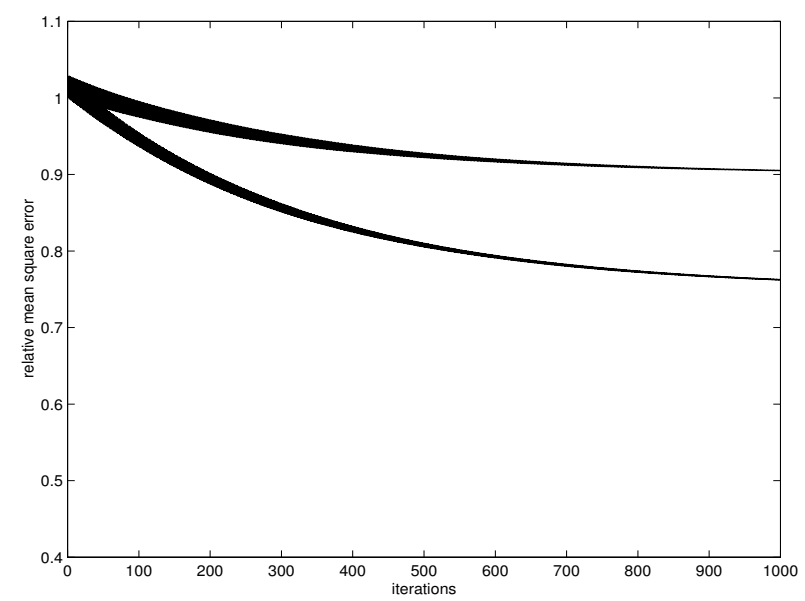

Fig. 4. RMSE of the SSH of the top and the lower layer. The lower curve is the RMSE for the surface layer while the upper curve is the RMSE for the second lower layer.

[3] A. Asif and J. M. F. Moura, "Data Assimilation in Large Time Varying Multidimensional Fields," IEEE Transactions on Image Processing, vol. 8, No. 11, Nov. 1999.

[4] D. R. Moore and A. J. Wallcraft, "Formulation of the NRL Layered Ocean Model in Spherical Coordinates," Tech. Rep. CR 7323-96-0005, Nav. Res. Lab., Stennis Space Center, MS, 1995.

[5] A. Asif and J. M. F. Moura, "Inversion of Block Matrices with L-Block Banded Inverse," Tech. Rep. Dept. Comp. Sc., York Univ., Toronto, ON, Dec. 2002, submitted for publication. 